\title{
Biophysical performance of different multipurpose trees species in Jharkhand, India
}

\author{
Bikash Das $^{1}$, Pradip Kumar Sarkar ${ }^{1, *}$, Neelam Kumari ${ }^{1}$, P. Dey ${ }^{2}$, A. K. Singh ${ }^{1}$ \\ and B. P. Bhatt ${ }^{3}$ \\ ${ }^{1}$ ICAR-Research Complex for Eastern Region, Research Centre, Ranchi 834 010, India \\ ${ }^{2}$ ICAR-Indian Institute of Soil Science, Bhopal 462 038, India \\ ${ }^{3}$ ICAR-Research Complex for Eastern Region, Patna 800 014, India
}

An experiment was conducted to evaluate the performance and characterize plant growth behaviour, light profile and soil fertility status of seventeen different trees (12 years old). The study indicated higher biomass production potential of Gmelina arborea, Dalbergia sissoo and Leucaena leucocephala. Higher photosynthetically active radiation value below tree canopy indicates compatibility of trees with a majority of agricultural crops. In the present study, a significant increase in $\mathrm{pH}$ and decrease in $\mathrm{EC}$ of trees were observed. The significant reduction in nutrients in different trees indicated a need for replenishment of nutrients in soil for maintaining soil fertility in agroforestry systems on long-term basis.

Keywords: Agroforestry, biophysical performance, CO2FIX model, multipurpose trees species, tree biomass.

THE physiography of Jharkhand state of India is characterized by light textured soil, undulating topography and high rate of soil erosion ${ }^{1}$. The agrarian scenario of the region is generally rainfed where rice-based monocropping is the prevalent practice. The soils of the region are low in organic carbon and available phosphorus content with deficiency of boron and zinc. Under these conditions, the productivity of majority of agricultural crops is low leading to unprofitability of upland agriculture ${ }^{2}$.

Soil without tree cover on hilly slope associated with more intensive agricultural practices is vulnerable to erosion and reduced fertility. Agroforestry can be one of the effective land-use management practices which can check land degradation ${ }^{3}$. The variety of products obtained from trees from agroforestry land-use practices are timber and other non-timber forest products such as fruits, seeds, bulbs, bark, fibres, roots, leaves, small wooden logs, firewood, etc. ${ }^{4,5}$. Integration of multipurpose trees in the existing production system can effectively improve the overall profitability of farming in Jharkhand. Being a tribal-dominated area, multipurpose tree species (MPTs) have always been an integral part of agriculture of the state. Trees like Gmelina arborea (Gamhar), Melia aze-

\footnotetext{
*For correspondence. (e-mail: pradipsarkar.tripura@gmail.com)
}

darach (Bakaine), Azadirachta indica (Neem), Terminalia arjuna (Arjun), Pongamia pinnata (Karanj), Dalbergia sissoo (Shisham), etc. are commonly found in the farm areas of tribal farmers ${ }^{6}$. However, limited information is available on the comparative growth performance ${ }^{7}$ and biomass production potential of different timer trees and MPTs in Jharkhand. Suitability of a tree species to be integrated with agricultural crops depends on light profile below the canopy and this information is also not available for the timber trees and MPTs. Improvement in soil fertility through integration of trees is recorded in many agroforestry systems ${ }^{8}$, though it is limited regarding the acidic soil of Jharkhand ${ }^{9}$. Keeping this in view, an experiment was initiated at ICAR-Research Complex for Eastern Region, Research Centre, Ranchi during 2003 to evaluate the performance of different multipurpose trees. In the present article, an effort has been made to characterize the growth behaviour of 12-year-old 17 tree species, light profile below the tree canopy and changes in soil fertility induced by the trees.

\section{Materials and methods}

The study was conducted at Ranchi, Jharkhand $\left(23^{\circ} 16^{\prime} 19.73^{\prime \prime} \mathrm{N}\right.$ and $85^{\circ} 20^{\prime} 49.99^{\prime \prime} \mathrm{E}$, altitude $\left.657.76 \mathrm{~m}\right)$ during 2014-15. Data on different plant growth parameters, and light profile were recorded from 12-year-old plants of 17 species including timber and MPTs. The plants were planted at a spacing of $5 \mathrm{~m} \times 5 \mathrm{~m}$ accommodating 400 plants per ha. Data were also recorded regarding the soil properties under different trees at depths of $0-15 \mathrm{~cm}$ and $15-30 \mathrm{~cm}$.

Tree height was measured using RAVI/Haga altimeter. Trunk circumference at breast height was measured using a measuring tape. To derive the volume of tree, we used diameter at breast height $(\mathrm{DBH})$ and height $(H)$ of the tree. We recorded data for four years, viz. 2007, 2012, 2014 and 2015, and calculated average tree volume $(V)$ of the particular tree species and current annual increment (CAI) using volume equations ${ }^{10}$, which were obtained by averaging the tree volumes $\left(V_{1}\right.$ and $\left.V_{2}\right)$ after considering trees as average of cylindrical and conical shape. 
Tree volume, $V_{1}\left(\mathrm{~m}^{3}\right.$ tree $\left.^{-1}\right)=\left(\pi \times \mathrm{DBH}^{2} \times H\right) / 4$

(for cylindrical shape).

Tree volume, $V_{2}\left(\mathrm{~m}^{3}\right.$ tree $\left.^{-1}\right)=\left(\pi \times \mathrm{DBH}^{2} \times H\right) / 12$

(for conical shape).

Thus

$$
V\left(\mathrm{~m}^{3} \operatorname{tree}^{-1}\right)=\left(V_{1}+V_{2}\right) / 2 .
$$

Other parameters like crown spread/radius ${ }^{11}$, crown height $^{12}$ and light intensity ${ }^{12}$ (measured in kLUX using LUX meter) were also measured. During data recording, light intensity was noted every minute and the mean of these was considered for final calculation of photosynthetically active radiation (PAR).

Leaf area $\left(\mathrm{LA} ; \mathrm{cm}^{2}\right)$ was estimated based on the measurements of leaf length and/or leaf width which is an indirect non-destructive method. Leaf length $(L)$ was measured from lamina tip to the point of petiole intersection along the midrib and width $(W)$ was measured at the widest point perpendicular to the midrib. The leaf can be described by a shape between an ellipse (0.78) and a triangle (0.50) of leaf length and width since actual leaf area is generally larger than a triangle but smaller than an ellipse $^{13}$. Specific leaf weight was calculated as the ratio of dry mass of leaf to leaf area. Leaf area index (LAI) of each species was estimated using a canopy analyser.

\section{Estimation of tree biomass (by using CO2FIX model)}

\section{Input parameters for CO2FIX model}

To quantify tree biomass of the MPTs, a dynamic carbon accounting model CO2FIX v3.1 was considered for assessing the baseline tree biomass and its simulated tree biomass over 20 years. The main parameters relevant to CO2FIX model are the cohort-wise values for the stemCAI $\left(\mathrm{m}^{3} \mathrm{ha}^{-1}\right.$ year $\left.^{-1}\right)$; relative growth of the foliage, branches, leaf and root with respect to the stem growth; turnover rates for foliage, branches and roots over the years; and climate data of the site, viz. annual precipitation $(\mathrm{mm})$ and monthly values of minimum and maximum temperatures $\left({ }^{\circ} \mathrm{C}\right)$ (Tables $\left.1-4\right)$. Other inputs to the model include initial surface soil organic carbon $(\mathrm{Mg} \mathrm{C} \mathrm{ha-1})$, rotation length for the tree species, percentage of carbon contents in different tree parts, wood density and initial values of baseline carbon $\left(\mathrm{Mg} \mathrm{Cha}^{-1}\right)$ in different tree parts $^{14}$.

Soil parameters such as $\mathrm{pH}$ and EC were measured using standard procedures. Content of organic carbon was measured by Walkley and Black rapid titration method. Available elements like nitrogen $(\mathrm{N})$ was determined by alkaline permanganate method, phosphorus (P) by Bray's method using ascorbic acid ${ }^{15}$ and potassium (K) was determined by flame photometer in soil solution prepared in an extraction solution of normal ammonium acetate $(\mathrm{pH} \mathrm{7.0})^{15}$.

\section{Statistical analysis}

The data was analysed using SYSTAT ${ }^{\circledR} 11$ Statistics II by SYSTAT Software Inc., USA, used for computing basic descriptive statistics.

\section{Results and discussion}

Data on different plant growth parameters of both timber tree species and MPTs are presented in Table 5. Among timber trees, the plant height ranged between $4.9 \mathrm{~m}$ (Dalbergia latifolia) and $13 \mathrm{~m}$ (D. sissoo) whereas among MPTs, it ranged between $3.5 \mathrm{~m}$ (Sapindus mukorossi) and $9.1 \mathrm{~m}$ (Leucaena leucocephala). With respect to trunk circumference at breast height, maximum values among timber species and MPTs were recorded as $70.3 \mathrm{~cm}(G$. arborea) and $96.6 \mathrm{~cm}$ (M. azedarach) respectively. The maximum tree volume was recorded in $G$. arborea (timber tree) and M. azedarach (MPTs). In Chhattisgarh, India, Naugraiya and $\mathrm{Puri}^{16}$ reported similar plant height of 7.5-year-old L. leucocephala although the value for $T$. arjuna was markedly lower than that in the present study. In Bijapur (Madhya Pradesh), Devarnavadagi and Murthy ${ }^{17}$ recorded slower growth rate of 3-year-old plants of D. sissoo than L. leucocephala in contrast to the present findings. Again, trunk diameter at breast height of Albizia procera was higher than that of $D$. sissoo, although the present study indicated statistically similar values in both the species. As the experimental sites are under the same agro-climatic conditions (Eastern Plateau and Hills), variation in agro-ecological conditions within the region might have contributed towards this contrasting result. Singh and Singh $^{7}$ also reported variation in plant growth parameters of different MPTs under different sub-zones of eastern plateau and hill agro-climatic conditions. The maximum canopy spread was recorded in $A$. procera (timber tree) and $M$. azedarach (MPTs). The minimum canopy spread was recorded in $P$. pinnata followed by Aegle marmelos, and $A$. indica which suggested the suitability of these species to be planted at a closer spacing under different agroforestry systems. The crown volume of $M$. azedarach was significantly higher than other MPTs. Among the timber species, crown volume was recorded maximum in $G$. arborea which was at par with A. procera. Crown volume was found to be significantly and positively correlated $(0.75)$ with trunk circumference. Hence, based on the overall plant growth parameters, $M$. azedarach, L. leucocephala, G. arborea and D. sissoo were found to be fast growing species; A. marmelos, $A$. procera, D. latifolia, Emblica officinalis, Pterocarpus marsupium, Tectona grandis and T. arjuna were found to 
Table 1. Parameters used in CO2FIX model for simulating tree biomass components in different tree cohorts

\begin{tabular}{|c|c|c|c|}
\hline \multirow[b]{2}{*}{ Parameters } & \multicolumn{3}{|c|}{ Cohorts } \\
\hline & $\begin{array}{c}\text { Slow growing } \\
\text { trees }^{\mathrm{a}}\end{array}$ & $\begin{array}{l}\text { Medium growing } \\
\text { trees }^{\mathrm{a}}\end{array}$ & $\begin{array}{c}\text { Fast growing } \\
\text { trees }^{\mathrm{a}}\end{array}$ \\
\hline Rotation years & 90 & 50 & 10 \\
\hline Wood density ( $\left.\mathrm{Mg} \mathrm{DM} \mathrm{m} \mathrm{m}^{-3}\right)$ & 0.67 & 0.65 & 0.61 \\
\hline Carbon content ( $\%$ dry weight) & 48 & 48 & 48 \\
\hline Turnover rate foliage & 0.5 & 0.5 & 0.6 \\
\hline Turnover rate branch & 0.02 & 0.04 & 0.02 \\
\hline Turnover rate root & 0.02 & 0.1 & 0.2 \\
\hline \multicolumn{4}{|c|}{ Product allocation for thinning harvesting ${ }^{\mathrm{a}}$} \\
\hline Stem log wood & 0.8 & 0.8 & 0.8 \\
\hline Stem slash & 0.2 & 0.2 & 0.2 \\
\hline Branch log wood & 0.8 & 0.8 & 0.2 \\
\hline Branch slash & 0.2 & 0.2 & 0.8 \\
\hline Foliage slash & 1 & 1 & 1 \\
\hline Foliage slash soil & 0.7 & 0.7 & 0.7 \\
\hline
\end{tabular}

${ }^{\mathrm{a}}$ Ajit et al. ${ }^{14}$.

Table 2. Estimated current annual increment (CAI) of the stem volume growth $\left(\mathrm{m}^{3} \mathrm{ha}^{-1}\right.$ year $\left.^{-1}\right)$ over years for three tree cohorts

\begin{tabular}{|c|c|c|c|c|c|}
\hline \multicolumn{2}{|c|}{ Slow growing } & \multicolumn{2}{|c|}{ Medium growing } & \multicolumn{2}{|c|}{ Fast growing } \\
\hline Age & $\mathrm{CAI}^{*}$ & Age & CAI & Age & CAI \\
\hline 0 & 4.94 & 0 & 8.00 & 0 & 10.67 \\
\hline 1 & 2.77 & 1 & 21.33 & 1 & 42.67 \\
\hline 2 & 2.51 & 2 & 34.67 & 2 & 88.00 \\
\hline 3 & 2.42 & 3 & 45.33 & 3 & 88.00 \\
\hline 4 & 2.41 & 4 & 56.00 & 4 & 101.33 \\
\hline 5 & 2.42 & 5 & 69.33 & 5 & 112.00 \\
\hline 6 & 2.47 & 6 & 77.33 & 6 & 122.67 \\
\hline 7 & 2.53 & 7 & 88.00 & 7 & 133.33 \\
\hline 8 & 1.29 & 8 & 93.33 & 8 & 136.00 \\
\hline 9 & 1.52 & 9 & 93.33 & 9 & 138.67 \\
\hline 10 & 2.47 & 10 & 96.00 & 10 & 138.67 \\
\hline
\end{tabular}

be medium growing species and $A$. indica, Madhuca integrifolia, $P$. pinnata and $S$. mukorossi were found to be slow growing species. It is worth noting that the species mostly known for the uses of their wood are fast growing whereas MPT species are found to be slow growing. Height of the first branch is the most important indicator of suitability of the species for high value timber purposes. Among the timber species evaluated, maximum value was recorded in case of D. sissoo.

Tree growth and development in agroforestry system primarily depend on two major factors, viz. total photosynthesis carried out by all trees in a stand and the fraction of photosynthate that is allocated to stemwood. The amount of foliage can be estimated indirectly by a range of non-destructive techniques ${ }^{18}$ whereas the photosynthetic rate is difficult to measure directly. Plant LA is an important determinant of light interception and conse- quently of transpiration, photosynthesis and plant development ${ }^{19}$. In the present study, highest LA was recorded in T. grandis (timber) and M. azedarach (MPTs) (Table 6). However, the maximum specific leaf weight was recorded in T. grandis (timber) and M. integrifolia (MPT). LAI plays an essential role in theoretical production eco$\log y^{11}$. It is an important parameter in the functioning of forests, controlling plant development and exchange of energy between vegetation and atmosphere ${ }^{20}$. In the present study, the value of LAI was maximum in $A$. procera (timber) and $M$. azedarach (MPT) (Table 6). LAI was found to be significantly correlated with crown volume (0.57) and canopy spread area (0.68).

PAR below the canopy was significantly higher in case of MPTs such as P. pinnata, Acacia catechu, P. marsupium, $M$. integrifolia, A. indica, S. mukorossi, E. officinalis and timber trees such as G. arborea, D. latifolia and $A$. procera which indicated their suitability to be integrated with majority of annual crops (Table 6). Interestingly, PAR below canopy was significantly and negatively correlated with the height of first branching $(-0.57)$. The maximum values of absorbed or reflected PAR $\left(\mathrm{PAR}_{\mathrm{a} / \mathrm{r}}\right)$ were recorded in $T$. grandis (timber species) and $M$. azedarach (MPTs). Waring et al. ${ }^{18}$ stated that, within a single tree, more exposed foliage absorbs more radiation and has higher photosynthetic rates than shaded foliage. High amount of $\mathrm{PAR}_{\mathrm{a} / \mathrm{r}}$ indicated high rate of photosynthesis leading to high rate of tree growth of these species.

Estimation of biomass estimates and through sequential harvesting are useful for quantifying net primary productivity and carbon-cycle ${ }^{14}$. CO2FIX has been extensively used for estimating biomass and changes in soil carbon stocks for forestry, agriculture and agroforestry projects. In our study, the tree biomass of different AFS was found to be in the range of $41.92 \mathrm{Mg} \mathrm{DM} \mathrm{ha}^{-1}$ (S. mukorossi) to 
Table 3. Relative growth of various tree components with respect to stem growth for tree cohorts (over years)

\begin{tabular}{|c|c|c|c|c|c|c|}
\hline \multirow[b]{2}{*}{ Parameters } & \multicolumn{2}{|c|}{ Slow growing ${ }^{\mathrm{a}}$} & \multicolumn{2}{|c|}{ Medium growing $^{\mathrm{a}}$} & \multicolumn{2}{|c|}{ Fast growing $^{\mathrm{a}}$} \\
\hline & Age & Rates & Age & Rates & Age & Rates \\
\hline \multirow[t]{8}{*}{ Foliage } & 0 & 1.00 & 1 & 0.26 & 0 & 0.30 \\
\hline & 10 & 0.50 & 5 & 0.63 & 2 & 0.44 \\
\hline & 20 & 0.73 & 15 & 0.50 & 3 & 0.40 \\
\hline & 30 & 0.64 & 20 & 0.38 & 4 & 0.38 \\
\hline & 40 & 1.02 & 25 & 0.32 & 5 & 0.37 \\
\hline & 50 & 1.12 & 30 & 0.50 & 6 & 0.32 \\
\hline & 60 & 0.98 & & & 7 & 0.56 \\
\hline & 70 & 0.91 & & & 8 & 0.58 \\
\hline \multirow[t]{8}{*}{ Branch } & 0 & 0.20 & 1 & 0.44 & 0 & 0.25 \\
\hline & 10 & 0.18 & 5 & 0.44 & 2 & 0.22 \\
\hline & 20 & 0.15 & 15 & 0.33 & 3 & 0.18 \\
\hline & 30 & 0.16 & 20 & 0.38 & 4 & 0.18 \\
\hline & 40 & 0.16 & 25 & 0.32 & 5 & 0.21 \\
\hline & 50 & 0.15 & 30 & 0.32 & 6 & 0.28 \\
\hline & 60 & 0.14 & & & 7 & 0.43 \\
\hline & 70 & 0.14 & & & 8 & 0.58 \\
\hline \multirow[t]{8}{*}{ Root } & 0 & 0.40 & 1 & 0.44 & 0 & 0.30 \\
\hline & 10 & 0.40 & 5 & 0.48 & 2 & 0.43 \\
\hline & 20 & 0.39 & 15 & 0.63 & 3 & 0.58 \\
\hline & 30 & 0.30 & 20 & 0.60 & 4 & 0.49 \\
\hline & 40 & 0.31 & 25 & 0.77 & 5 & 0.36 \\
\hline & 50 & 0.31 & 30 & 0.82 & 6 & 0.31 \\
\hline & 60 & 0.29 & & & 7 & 0.47 \\
\hline & 70 & 0.27 & & & 8 & 0.37 \\
\hline
\end{tabular}

${ }^{\mathrm{a}} \mathrm{Ajit}$ et $a l .{ }^{14}$.

Table 4. Climate data of the study area

\begin{tabular}{lcccc}
\hline & \multicolumn{2}{c}{ Temperature $\left({ }^{\circ} \mathrm{C}\right)$} & & \multicolumn{2}{c}{$\begin{array}{c}\text { Rainfall } \\
\text { Month }\end{array}$} & Minimum & Maximum & during $2014-15(\mathrm{~mm})$ & Normal rainfall (mm)* \\
\cline { 2 - 4 } March & 9.4 & 23.4 & 0.0 & 25.0 \\
April & 11.9 & 27.8 & 38.0 & 21.8 \\
May & 15.1 & 30.7 & 67.0 & 61.7 \\
June & 20.6 & 32.8 & 291.0 & 249.4 \\
July & 24.0 & 37.3 & 302.0 & 336.6 \\
August & 25.8 & 33.8 & 189.0 & 319.1 \\
September & 25.1 & 30.0 & 45.0 & 247.3 \\
October & 24.9 & 29.9 & 56.0 & 76.6 \\
November & 23.2 & 31.4 & 56.0 & 10.8 \\
December & 18.8 & 30.3 & 5.0 & 16.7 \\
January & 14.6 & 27.9 & 8.0 & 21.2 \\
February & 10.8 & 24.8 & 5.0 & 16.7 \\
\hline
\end{tabular}

*From past recorded data of the institute (ICAR RCER, RC, Ranchi).

$190.98 \mathrm{Mg} \mathrm{DM} \mathrm{ha}^{-1}$ (G. arborea) (Table 7). When simulated, the tree biomass of the respective AFS over a simulated period of 20 years was in the range of $134.91 \mathrm{Mg}$ $\mathrm{DM} \mathrm{ha}^{-1}$ (S. mukorossi) to $469.39 \mathrm{Mg} \mathrm{DM} \mathrm{ha}^{-1}$ (G. arborea as fast growing timber tree). The biomass yield of $D$. sissoo and L. leucocephala was also significantly higher than other species. Onyekwelu ${ }^{21}$ also reported high biomass yield of $G$. arborea plantations (5-21 years ages) in Nigeria (83.2 tonne $\mathrm{ha}^{-1}$ in 5-year-old plant to 394.9 tonne $\mathrm{ha}^{-1}$ in 21-year-old plants). For degraded red lateritic soils in Central India, Swami et al. ${ }^{22}$ recorded stand biomass yield of $G$. arborea ranging from 3.94 (1-year-old) to $53.67 \mathrm{Mg} \mathrm{ha}^{-1}$ (6-year-old). Tree biomass was significantly and positively correlated with $\mathrm{PAR}_{\mathrm{a} / \mathrm{r}}$ (0.59) and canopy volume (0.75) which indicated more photosynthetic activity contributing towards tree biomass. 
Table 5. Plant canopy characteristics of different trees

\begin{tabular}{|c|c|c|c|c|c|}
\hline Species & $\begin{array}{l}\text { Tree height } \\
\text { (m) }\end{array}$ & $\begin{array}{l}\text { Trunk circumference } \\
\text { at breast height }(\mathrm{cm})\end{array}$ & $\begin{array}{c}\text { Canopy spread } \\
\text { area }\left(\mathrm{m}^{2}\right)\end{array}$ & $\begin{array}{l}\text { Average crown } \\
\text { volume }\left(\mathrm{m}^{3}\right)\end{array}$ & $\begin{array}{c}\text { Height up to } \\
\text { first branch }(\mathrm{m})\end{array}$ \\
\hline \multicolumn{6}{|l|}{ Timber trees } \\
\hline Albizia procera (Roxb.) Benth & $6.3 \pm 0.53^{d}$ & $53.0 \pm 6.11^{\mathrm{b}}$ & $37.59 \pm 2.73^{\mathrm{a}}$ & $111.1 \pm 15.47^{\mathrm{a}}$ & $1.9 \pm 0.15^{\mathrm{de}}$ \\
\hline Dalbergia latifolia Roxb. & $4.9 \pm 0.20^{\mathrm{e}}$ & $43.3 \pm 5.81^{\mathrm{d}}$ & $13.53 \pm 1.81^{\mathrm{d}}$ & $31.7 \pm 1.73^{\mathrm{d}}$ & $1.3 \pm 0.02^{\mathrm{e}}$ \\
\hline Dalbergia sissoo Roxb. & $13.0 \pm 0.76^{\mathrm{a}}$ & $58.3 \pm 3.18^{\mathrm{b}}$ & $16.63 \pm 1.26^{\mathrm{c}}$ & $91.3 \pm 5.59^{\mathrm{b}}$ & $4.7 \pm 0.43^{\mathrm{a}}$ \\
\hline Gmelina arborea Roxb. & $10.2 \pm 0.33^{\mathrm{b}}$ & $70.3 \pm 4.17^{\mathrm{a}}$ & $22.07 \pm 0.26^{\mathrm{b}}$ & $112.3 \pm 3.92^{\mathrm{a}}$ & $2.5 \pm 0.07^{\mathrm{c}}$ \\
\hline Tectona grandis Linn. & $6.9 \pm 0.13^{\mathrm{d}}$ & $46.3 \pm 2.40^{\mathrm{cd}}$ & $14.98 \pm 1.48^{\mathrm{c}}$ & $39.6 \pm 2.12^{\mathrm{d}}$ & $3.0 \pm 0.13^{\mathrm{b}}$ \\
\hline \multicolumn{6}{|l|}{ Multipurpose tree species (MPTS) } \\
\hline Acacia catechu (L.f) Willd. & $6.3 \pm 1.60^{\mathrm{b}}$ & $54.6 \pm 16.17^{\mathrm{c}}$ & $21.4 \pm 2.35^{\mathrm{c}}$ & $24.6 \pm 2.89^{\mathrm{e}}$ & $1.6 \pm 0.45^{\mathrm{ef}}$ \\
\hline Aegle marmelos (L.) Corrêa. & $4.6 \pm 0.17^{\mathrm{e}}$ & $44.6 \pm 6.06^{\mathrm{d}}$ & $3.47 \pm 0.38^{\mathrm{g}}$ & $5.4 \pm 0.88^{\mathrm{gh}}$ & $2.3 \pm 0.34^{\mathrm{c}}$ \\
\hline Azadirachta indica A. Juss. & $5.1 \pm 0.04^{\mathrm{d}}$ & $45.1 \pm 4.04^{\mathrm{d}}$ & $3.86 \pm 0.38^{\mathrm{fg}}$ & $7.1 \pm 1.22^{\mathrm{g}}$ & $2.3 \pm 0.51^{\mathrm{c}}$ \\
\hline Emblica officinalis Gaertn. & $5.7 \pm 0.21^{\mathrm{cd}}$ & $67.3 \pm 7.21^{\mathrm{b}}$ & $9.35 \pm 0.98^{\mathrm{d}}$ & $27.2 \pm 1.19^{\mathrm{e}}$ & $1.3 \pm 0.03^{\mathrm{g}}$ \\
\hline Leucaena leucocephala (Lam.) de Wit. & $9.1 \pm 0.17^{\mathrm{a}}$ & $47.6 \pm 4.48^{\mathrm{cd}}$ & $27.35 \pm 2.25^{\mathrm{b}}$ & $119.7 \pm 4.74^{\mathrm{b}}$ & $2.5 \pm 0.27^{\mathrm{ab}}$ \\
\hline Melia azedarach L. & $9.0 \pm 0.76^{\mathrm{a}}$ & $96.6 \pm 18.76^{\mathrm{a}}$ & $40.36 \pm 4.55^{\mathrm{a}}$ & $167.7 \pm 13.21^{\mathrm{a}}$ & $2.7 \pm 0.27^{\mathrm{a}}$ \\
\hline Pongamia pinnata (L.) Pierrre & $4.1 \pm 0.32^{\mathrm{e}}$ & $23.0 \pm 1.15^{\mathrm{f}}$ & $1.87 \pm 0.15^{\mathrm{h}}$ & $2.0 \pm 0.27^{\mathrm{h}}$ & $2.5 \pm 0.15^{\mathrm{ab}}$ \\
\hline Pterocarpus marsupium Roxb. & $5.8 \pm 0.15^{\mathrm{c}}$ & $52.3 \pm 1.45^{\mathrm{c}}$ & $26.89 \pm 2.78^{b}$ & $76.6 \pm 2.92^{\mathrm{c}}$ & $1.5 \pm 0.05^{\mathrm{f}}$ \\
\hline Sapindus mukorossi Gaertn. & $3.5 \pm 0.28^{\mathrm{f}}$ & $26.3 \pm 4.91^{\mathrm{f}}$ & $7.80 \pm 0.93^{\mathrm{e}}$ & $11.3 \pm 1.80^{\mathrm{f}}$ & $0.8 \pm 0.06^{\mathrm{h}}$ \\
\hline Terminalia arjuna (Roxb. ex DC.) Wight \& Arn & $6.4 \pm 0.30^{\mathrm{b}}$ & $53.0 \pm 8.14^{\mathrm{c}}$ & $17.11 \pm 1.53^{\mathrm{c}}$ & $49.0 \pm 4.31^{\mathrm{d}}$ & $2.1 \pm 0.10^{\mathrm{d}}$ \\
\hline
\end{tabular}

Values are represented as mean \pm SE. Values having different superscripts within rows are significantly different at $P<0.05$.

Table 6. Foliar characteristics and light profile of different trees

\begin{tabular}{|c|c|c|c|c|c|}
\hline Species & Leaf area $\left(\mathrm{cm}^{2}\right)$ & Leaf area index & $\begin{array}{l}\text { Specific leaf fresh } \\
\text { wt }\left(\mathrm{mg} \mathrm{cm}^{2}\right)\end{array}$ & $\begin{array}{l}\text { PAR }\left(\mu \mathrm{mol} \mathrm{cm}{ }^{-1}\right) \\
\text { below tree canopy }\end{array}$ & $\begin{array}{l}\text { PAR absorbed/ } \\
\text { reflected }\left(\mu \mathrm{mol} \mathrm{cm}{ }^{-1}\right)\end{array}$ \\
\hline \multicolumn{6}{|l|}{ Timber trees } \\
\hline A. procera & $366.70 \pm 6.36^{\mathrm{b}}$ & $10.54 \pm 2.13^{\mathrm{a}}$ & $0.19 \pm 0.02^{\mathrm{d}}$ & $829.16 \pm 123.99^{\mathrm{a}}$ & $944.07 \pm 69.13^{\mathrm{b}}$ \\
\hline D. latifolia & $65.89 \pm 1.18^{\mathrm{d}}$ & $1.16 \pm 0.05^{\mathrm{c}}$ & $3.94 \pm 0.57^{\mathrm{c}}$ & $977.33 \pm 66.89^{\mathrm{a}}$ & $795.93 \pm 51.07^{\mathrm{c}}$ \\
\hline D. sissoo & $58.84 \pm 1.18^{\mathrm{d}}$ & $0.69 \pm 0.11^{\mathrm{d}}$ & $4.8 \pm 0.67^{\mathrm{c}}$ & $316.54 \pm 16.67^{b}$ & $1456.67 \pm 111.18^{\mathrm{a}}$ \\
\hline G. arborea & $170.80 \pm 4.03^{c}$ & $0.53 \pm 0.03^{\mathrm{d}}$ & $12.0 \pm 4.69^{\mathrm{b}}$ & $996.08 \pm 343.23^{\mathrm{a}}$ & $728.70 \pm 79.06^{\mathrm{c}}$ \\
\hline T. grandis & $791.91 \pm 11.40^{\mathrm{a}}$ & $0.64 \pm 0.06^{\mathrm{d}}$ & $15.35 \pm 3.22^{\mathrm{a}}$ & $235.76 \pm 64.99^{\mathrm{b}}$ & $1537.41 \pm 128.18^{\mathrm{a}}$ \\
\hline \multicolumn{6}{|l|}{ MPTS } \\
\hline A. catechu & $11.46 \pm 2.16^{\mathrm{e}}$ & $0.34 \pm 0.06^{\mathrm{f}}$ & $0.25 \pm 0.04^{\mathrm{h}}$ & $794.43 \pm 78.37^{\mathrm{bc}}$ & $984.36 \pm 99.13^{\mathrm{b}}$ \\
\hline A. marmelos & $131.05 \pm 0.81^{\mathrm{c}}$ & $2.48 \pm 0.36^{\mathrm{c}}$ & $3.12 \pm 0.27^{\mathrm{e}}$ & $243.33 \pm 6.68^{f}$ & $1529.81 \pm 15.16^{\mathrm{a}}$ \\
\hline A. indica & $180.54 \pm 2.5^{\mathrm{b}}$ & $7.11 \pm 0.78^{\mathrm{b}}$ & $0.36 \pm 0.03^{\mathrm{h}}$ & $873.16 \pm 376.65^{\mathrm{b}}$ & $900.00 \pm 8.14^{\mathrm{c}}$ \\
\hline E. officinalis & $1.80 \pm 0.11^{\mathrm{f}}$ & $0.27 \pm 0.06^{\mathrm{f}}$ & $21.17 \pm 2.07^{\mathrm{b}}$ & $796.08 \pm 131.37^{\mathrm{c}}$ & $977.04 \pm 67.08^{\mathrm{cd}}$ \\
\hline L. leucocephala & $70.21 \pm 2.34^{\mathrm{d}}$ & $1.71 \pm 0.14^{\mathrm{d}}$ & $0.95 \pm 0.15^{\mathrm{g}}$ & $588.54 \pm 125.04^{\mathrm{d}}$ & $1023.70 \pm 110.10^{\mathrm{b}}$ \\
\hline M. azedarach & $290.21 \pm 4.38^{\mathrm{a}}$ & $15.55 \pm 4.42^{\mathrm{a}}$ & $0.14 \pm 0.05^{\mathrm{h}}$ & $284.38 \pm 36.83^{\mathrm{e}}$ & $1535.37 \pm 151.15^{\mathrm{a}}$ \\
\hline$P$. pinnata & $54.89 \pm 0.49^{\mathrm{de}}$ & $0.70 \pm 0.01^{\mathrm{e}}$ & $8.6 \pm 0.66^{\mathrm{de}}$ & $1192.97 \pm 260.05^{\mathrm{a}}$ & $580.19 \pm 63.04^{\mathrm{d}}$ \\
\hline P. marsupium & $199.91 \pm 2.85^{b}$ & $8.32 \pm 1.86^{\mathrm{b}}$ & $2.32 \pm 0.21^{\mathrm{f}}$ & $1033.17 \pm 105.34^{\mathrm{a}}$ & $740.00 \pm 81.06^{\mathrm{d}}$ \\
\hline S. mukorossi & $29.66 \pm 1.27^{\mathrm{e}}$ & $0.33 \pm 0.05^{\mathrm{f}}$ & $14.3 \pm 1.57^{\mathrm{c}}$ & $1102.54 \pm 136.44^{\mathrm{a}}$ & $670.56 \pm 53.05^{\mathrm{e}}$ \\
\hline T. arjuna & $55.74 \pm 0.43^{d}$ & $0.33 \pm 0.09^{\mathrm{f}}$ & $9.29 \pm 1.49^{\mathrm{d}}$ & $377.67 \pm 127.25^{\mathrm{e}}$ & $1395.56 \pm 128.16^{\mathrm{ab}}$ \\
\hline
\end{tabular}

Values are represented as mean \pm SE. Values having different superscripts within rows are significantly different at $P<0.05$.

Significant variation in soil properties was recorded for different trees (Table 8). Although the soil $\mathrm{pH}$ at $0-15 \mathrm{~cm}$ depth did not vary significantly, at $15-30 \mathrm{~cm}$ depth it was significantly higher than that in controlled condition under M. azedarach, D. sissoo, T. grandis, A. procera, M. integrifolia, T. arjuna, $P$. pinnata, $G$. arborea, L. leucocephala, S. robusta. Imayavarambar et al. ${ }^{23}$ and Oraon et al. ${ }^{9}$ also reported improving trend of soil $\mathrm{pH}$ under different agroforestry species. With respect to elec- trical conductivity, significant decrease at $0-15 \mathrm{~cm}$ was recorded for $A$. indica and the value did not change significantly for $T$. arjuna, $E$. officinalis, $P$. pinnata, G. arborea, L. leucocephala and S. robusta. At $15-30 \mathrm{~cm}$ soil depth, maximum EC was recorded for D. sissoo which was at par with that of $M$. azedarach. The soil organic carbon did not change significantly for different species. With respect to available $\mathrm{N}$, the value at $0-15 \mathrm{~cm}$ depth decreased significantly for $T$. grandis, D. latifolia 
Table 7. Species-wise tree biomass accumulation in different trees (simulated over 20 years using CO2FIX model)

\begin{tabular}{lcc}
\hline & Tree biomass (above and below ground) Mg DM ha ${ }^{-1}$ \\
Species & Base line & Simulated \\
\cline { 2 - 3 } Slow growing & & \\
A. indica & 49.54 & 142.53 \\
M. integrifolia & 57.90 & 150.89 \\
$P$. pinnata & 46.94 & 139.93 \\
S. mukorossi & 41.92 & 134.91 \\
Medium growing & & \\
A. marmelos & 143.07 & 384.51 \\
A. procera & 141.09 & 382.53 \\
D. latifolia & 141.09 & 382.53 \\
S. robusta & 136.42 & 363.90 \\
E. officinalis & 130.07 & 361.00 \\
$P$. marsupium & 145.05 & 386.49 \\
T. grandis & 137.29 & 378.72 \\
T. arjuna & 149.16 & 390.60 \\
A. catechu & 121.36 & 342.18 \\
Fast growing & & \\
D. sissoo & 172.47 & 450.88 \\
G. arborea & 190.98 & 469.39 \\
L. leucocephala & 172.47 & 450.88 \\
M. azedarach & 168.30 & 446.71 \\
\hline
\end{tabular}

Table 8. Soil properties under different trees

\begin{tabular}{|c|c|c|c|c|c|c|c|c|}
\hline \multirow[b]{2}{*}{ Species } & \multicolumn{2}{|c|}{$\mathrm{pH}$} & \multicolumn{2}{|c|}{$\mathrm{EC}$} & \multicolumn{2}{|c|}{ Available N } & \multicolumn{2}{|c|}{ Available $\mathrm{K}_{2} \mathrm{O}$} \\
\hline & $\begin{array}{c}0-15 \mathrm{~cm} \\
\text { depth }\end{array}$ & $\begin{array}{c}15-30 \mathrm{~cm} \\
\text { depth }\end{array}$ & $\begin{array}{c}0-15 \mathrm{~cm} \\
\text { depth }\end{array}$ & $\begin{array}{c}15-30 \mathrm{~cm} \\
\text { depth }\end{array}$ & $\begin{array}{c}0-15 \mathrm{~cm} \\
\text { depth }\end{array}$ & $\begin{array}{c}15-30 \mathrm{~cm} \\
\text { depth }\end{array}$ & $\begin{array}{l}0-15 \mathrm{~cm} \\
\text { depth }\end{array}$ & $\begin{array}{c}15-30 \mathrm{~cm} \\
\text { depth }\end{array}$ \\
\hline A. marmelos & $5.53 \pm 0.29^{\mathrm{a}}$ & $5.32 \pm 0.16^{\mathrm{b}}$ & $0.06 \pm 0.005^{\mathrm{a}}$ & $0.07 \pm 0.01^{\mathrm{b}}$ & $406.9 \pm 26.1^{\mathrm{a}}$ & $427.4 \pm 45.9^{\mathrm{a}}$ & $182.4 \pm 22.1^{\mathrm{b}}$ & $265.6 \pm 86.5^{\mathrm{a}}$ \\
\hline A. procera & $5.53 \pm 0.24^{\mathrm{a}}$ & $5.58 \pm 0.22^{\mathrm{a}}$ & $0.08 \pm 0.006^{\mathrm{a}}$ & $0.07 \pm 0.020^{\mathrm{b}}$ & $418.1 \pm 22.0^{\mathrm{a}}$ & $472.3 \pm 19.7^{\mathrm{a}}$ & $275.2 \pm 53.2^{\mathrm{a}}$ & $212.8 \pm 33.3^{b}$ \\
\hline A. indica & $5.20 \pm 0.09^{\mathrm{a}}$ & $5.27 \pm 0.09^{\mathrm{b}}$ & $0.01 \pm 0.003^{\mathrm{d}}$ & $0.02 \pm 0.001^{\mathrm{d}}$ & $434.9 \pm 7.4^{\mathrm{a}}$ & $389.2 \pm 33.9^{\mathrm{a}}$ & $267.2 \pm 47.1^{\mathrm{a}}$ & $297.6 \pm 8.3^{\mathrm{a}}$ \\
\hline D. sissoo & $5.59 \pm 0.35^{\mathrm{a}}$ & $5.47 \pm 0.01^{\mathrm{a}}$ & $0.07 \pm 0.017^{\mathrm{a}}$ & $0.12 \pm 0.020^{\mathrm{a}}$ & $399.4 \pm 11.3^{\mathrm{a}}$ & $380.8 \pm 29.1^{\mathrm{a}}$ & $164.8 \pm 23.9^{\mathrm{b}}$ & $187.2 \pm 2.7^{\mathrm{b}}$ \\
\hline E. officinalis & $5.12 \pm 0.09^{\mathrm{a}}$ & $5.20 \pm 0.07^{\mathrm{b}}$ & $0.02 \pm 0.001^{\mathrm{c}}$ & $0.02 \pm 0.001^{\mathrm{d}}$ & $390.1 \pm 22.0^{\mathrm{a}}$ & $358.4 \pm 8.5^{\mathrm{a}}$ & $267.2 \pm 14.2^{\mathrm{a}}$ & $281.6 \pm 5.7^{\mathrm{a}}$ \\
\hline G. arborea & $5.48 \pm 0.20^{\mathrm{a}}$ & $5.65 \pm 0.16^{\mathrm{a}}$ & $0.02 \pm 0.003^{\mathrm{c}}$ & $0.02 \pm 0.001^{\mathrm{d}}$ & $401.3 \pm 9.3^{\mathrm{a}}$ & $380.8 \pm 28.7^{\mathrm{a}}$ & $387.4 \pm 31.2^{\mathrm{a}}$ & $294.4 \pm 61.0^{\mathrm{a}}$ \\
\hline L. leucocephala & $5.56 \pm 0.09^{\mathrm{a}}$ & $5.69 \pm 0.15^{\mathrm{a}}$ & $0.02 \pm 0.003^{c}$ & $0.02 \pm 0.003^{\mathrm{d}}$ & $423.7 \pm 26.1^{\mathrm{a}}$ & $405.1 \pm 27.1^{\mathrm{a}}$ & $302.4 \pm 84.9^{\mathrm{a}}$ & $254.4 \pm 49.9^{\mathrm{a}}$ \\
\hline M. integrifolia & $5.07 \pm 0.04^{\mathrm{a}}$ & $5.53 \pm 0.23^{\mathrm{a}}$ & $0.05 \pm 0.005^{\mathrm{b}}$ & $0.05 \pm 0.004^{\mathrm{c}}$ & $402.5 \pm 23.4^{\mathrm{a}}$ & $417.2 \pm 11.3^{\mathrm{a}}$ & $156.8 \pm 16.2^{\mathrm{b}}$ & $168.0 \pm 30.5^{\mathrm{b}}$ \\
\hline M. azedarach & $5.84 \pm 0.56^{\mathrm{a}}$ & $6.15 \pm 0.43^{\mathrm{a}}$ & $0.07 \pm 0.020^{\mathrm{a}}$ & $0.09 \pm 0.035^{\mathrm{a}}$ & $293.0 \pm 33.2^{\mathrm{c}}$ & $393.8 \pm 19.7^{\mathrm{a}}$ & $177.6 \pm 45.6^{\mathrm{b}}$ & $305.6 \pm 79.6^{\mathrm{a}}$ \\
\hline P. pinnata & $5.09 \pm 0.02^{\mathrm{a}}$ & $5.55 \pm 0.02^{\mathrm{a}}$ & $0.02 \pm 0.002^{c}$ & $0.02 \pm 0.001^{\mathrm{d}}$ & $386.4 \pm 25.8^{\mathrm{a}}$ & $389.2 \pm 4.8^{\mathrm{a}}$ & $360.0 \pm 5.5^{\mathrm{a}}$ & $367.2 \pm 37.4^{\mathrm{a}}$ \\
\hline P. marsupium & $5.19 \pm 0.09^{\mathrm{a}}$ & $5.28 \pm 0.08^{\mathrm{b}}$ & $0.02 \pm 0.001^{\mathrm{c}}$ & $0.02 \pm 0.001^{\mathrm{d}}$ & $379.1 \pm 23.38^{\mathrm{a}}$ & $395.6 \pm 24.4^{\mathrm{a}}$ & $220.5 \pm 34.3^{b}$ & $231.2 \pm 36.0^{\mathrm{b}}$ \\
\hline S. robusta & $5.02 \pm 0.01^{\mathrm{a}}$ & $5.67 \pm 0.09^{\mathrm{a}}$ & $0.02 \pm 0.002^{\mathrm{c}}$ & $0.01 \pm 0.001^{\mathrm{d}}$ & $400.4 \pm 1.6^{\mathrm{a}}$ & $417.2 \pm 24.2^{\mathrm{a}}$ & $218.4 \pm 34.6^{b}$ & $235.2 \pm 5.5^{\mathrm{b}}$ \\
\hline T. grandis & $5.39 \pm 0.14^{\mathrm{a}}$ & $5.48 \pm 0.09^{\mathrm{a}}$ & $0.04 \pm 0.01^{b}$ & $0.05 \pm 0.014^{\mathrm{c}}$ & $368.1 \pm 29.5^{\mathrm{b}}$ & $459.2 \pm 25.2^{\mathrm{a}}$ & $171.4 \pm 20.2^{b}$ & $180.8 \pm 20.8^{b}$ \\
\hline T. arjuna & $5.47 \pm 0.07^{\mathrm{a}}$ & $5.55 \pm 0.05^{\mathrm{a}}$ & $0.02 \pm 0.003^{\mathrm{c}}$ & $0.02 \pm 0.003^{\mathrm{d}}$ & $382.6 \pm 21.0^{\mathrm{a}}$ & $429.3 \pm 31.9^{\mathrm{a}}$ & $288.0 \pm 93.6^{\mathrm{a}}$ & $337.6 \pm 34.2^{\mathrm{a}}$ \\
\hline Fallow & $5.32 \pm 0.12^{\mathrm{a}}$ & $5.30 \pm 0.01^{\mathrm{b}}$ & $0.03 \pm 0.003^{c}$ & $0.02 \pm 0.003^{\mathrm{d}}$ & $388.2 \pm 29.3^{\mathrm{a}}$ & $375.2 \pm 19.4^{\mathrm{a}}$ & $276.8 \pm 15.2^{\mathrm{a}}$ & $276.0 \pm 29.1^{\mathrm{a}}$ \\
\hline
\end{tabular}

Values are represented as mean \pm SE. Values having different superscripts within rows are significantly different at $P<0.05$.

and $M$. azedarach. However, at $15-30 \mathrm{~cm}$ depth, no significant change in available $\mathrm{N}$ was recorded. The available $\mathrm{P}$ content also did not change significantly at both the soil depths. In case of available $\mathrm{K}$, significant reduction than the fallow was recorded at $0-15 \mathrm{~cm}$ soil depth for $A$. marmelos, M. azedarach, D. latifolia, D. sissoo, T. grandis, $M$. integrifolia and $S$. mukorossi. At $15-30 \mathrm{~cm}$ depth, value reduction was recorded for $D$. latifolia, $D$. sissoo,
T. grandis, M. integrifolia and S. mukorossi. Shepherd et $a l .{ }^{8}$ found that agroforestry systems did not significantly reduce $\mathrm{N}$ deficits except when a high proportion of the total biomass was returned to the soil, rather than removed from the farm. Although agroforestry increased $\mathrm{N}$ input through biological $\mathrm{N}$ fixation and deep $\mathrm{N}$ uptake, this was offset by a larger nutrient removal from the farm in harvested products. Hence, reduction in the available $\mathrm{N}$ 


\section{RESEARCH ARTICLES}

and $\mathrm{K}$ content in the soil in our study can be attributed to higher uptake by the species and variation in soil depth can be attributed to variation in feeder root distribution among the species. This warrants additional nutrient supplementation for acid soils of eastern plateau and hill conditions of India for maintaining soil fertility on a long-term basis.

\section{Conclusion}

This article aimed at characterizing the different treebased (i.e. timber trees and MPTs) agroforestry systems considering the tree canopy and plant growth and development parameters. The study clearly indicated higher biomass production potential of G. arborea, D. sissoo and L. leucocephala in Jharkhand. The study also showed higher PAR below tree canopy for P. pinnata, A. catechu, P. marsupium, M. integrifolia, A. indica, S. mukorossi, E. officinalis, G. arborea, D. latifolia and A. procera indicating their compatibility with majority of the agricultural crops. Finally there is a need for replenishment of nutrients in the soil for long-term sustainability of soil fertility in agroforestry systems.

1. Gulati, A. and Rai, S. C., Cost estimation of soil erosion and nutrient loss from a watershed of the Chotanagpur Plateau, India, Curr. Sci., 2014, 107, 670-674.

2. Dey, P. and Sarkar, A. K., Revisiting indigenous farming knowledge of Jharkhand (India) for conservation of natural resources and combating climate change. Indian J. Tradit. Knowl., 2011, 10, 71-79.

3. Ehui, S. K., Kang, B. T. and Spencer, D. S. C., Economic analysis of soil erosion effects in alley cropping, no-till and bush fallow systems in South Western Nigeria. Agric. Syst., 1990, 34, 349368 .

4. Cunningham, A. B., People, park and plant use. Recommendations for multiple use zones and development alternatives around Bwindi Impenetrable National Park, Uganda, UNESCO, Paris, 1996.

5. Peters, C. M., Sustainable harvest of non-timber plant resources in tropical moist forest: an ecological primer, USAID Biodiversity Support Programme, Washington DC, 1994.

6. Yadava, M. S. and Quli, S. M. S., Agroforestry systems and practices in Jharkhand. In Agroforestry Systems and Practices (eds Puri, S. and Panwar, P.), New India Publishing Agency, New Delhi, 2007, pp. 305-318.

7. Singh, M. P. and Singh, S., Growth performance of timber species under forest plantations in different agro-climatic zone of Jharkhand, India. Indian For., 2015, 141, 819-825.

8. Shepherd, K. D., Ohlsson, E., Okalebo, J. R. and Ndufa, J. K., Potential impact of agroforestry on soil nutrient balances at the farm scale in the East African Highlands. Fert. Res., 1995, 44, 8799

9. Oraon, B. C., Malik, M. S. and Bijalwan, A., Changes in soil properties under plantation of multipurpose trees in different ecosystems of Jharkhand. Appl. Ecol. Environ. Sci., 2014, 2, 110113.

10. Ravindranath, N. H. and Ostwald, M., Carbon inventory methods: handbook for greenhouse gas inventory, carbon mitigation and round wood production projects (Advance in Global Change Research-29), Springer Science, Dordrecht, 2008, p. 306.

11. Breda, N., Ground-based measurements of leaf area index: a review of methods, instruments and current controversies. J. Exp. Bot., 2003, 54, 2403-2417.

12. Evans, M. R. et al., Allometry and growth of eight tree taxa in United Kingdom woodlands. Sci. Data, Nature, 2015; doi:10.1038/sdata.2015.6.

13. Zhang, L. and Liu, X., Non-destructive leaf-area estimation for Bergenia purpurascens across timberline ecotone, southeast Tibet. Ann. Bot. Fennici, 2010, 47, 346-352.

14. Ajit, S. K. et al., Modeling analysis of potential carbon sequestration under existing agroforestry systems in three districts of Indo-gangetic plains in India. Agroforest. Sys., 2013, 87, 213225 .

15. Tandon, H. L. S., Methods of Analysis of Soils, Plants, Waters and Fertilizers, FDCO, New Delhi, 1999.

16. Naugraiya, M. N. and Puri, S., Performance of multipurpose tree species under agroforestry systems on Entisols of Chattisgarh plains. Range Manage. Agrofor., 2001, 22, 164-172.

17. Devarnavadagi, S. B. and Murthy, B. G., Performance of different tree species on eroded soils of northern dry zone of Karnataka. In Advances in Agricultural Research in India, 1995, vol. 4, pp. 7377.

18. Waring, R. H., Schroeder, P. E. and Oren, R., Application of the pipe model theory to predict leaf area. Can. J. For. Res., 1982, 12, 556-560.

19. Goudriaan, J. and Van Laar, H. H., Modeling Potential Crop Growth Processes, Kluwer, Dordrecht, The Netherlands, 1994.

20. Moser, G., Hertel, D. and Leuschner, C., Altitudinal change in LAI and stand leaf biomass in tropical Montane forests: a transect study in Ecuador and a Pan-Tropical Meta-Analysis. Ecosyst., 2007, 198, 229-242.

21. Onyekwelu, J. C., Above-ground biomass production and biomass equations for even-aged Gmelina arborea (Roxb) plantations in south-western Nigeria. Biomass and Bioenergy, 2004, 26, 39-46.

22. Swamy, S. L., Puri, S. and Singh, A. K., Growth, biomass, carbon storage and nutrient distribution in Gmelina arborea Roxb. stands on red lateritic soils in central India. Bioresource Technol., 2003, 90, 109-126.

23. Imayavarambar, V., Singaravel, R., Thanunathan, K. and Kardasamy, Study on the soil fertility enrichment under Levcaena leuescephata plantation. Ind. J. For., 2001, 24, 478-479.

Received 11 January 2016; revised accepted 26 August 2018

doi: $10.18520 / \mathrm{cs} / \mathrm{v} 116 / \mathrm{i} 1 / 82-88$ 